\title{
Transcriptome sequencing and analysis reveals the molecular response to selenium stimuli in Pueraria lobata(willd.) Ohwi
}

\author{
Kunyuan Guo ${ }^{1}$, Yiwei Yao ${ }^{2}$, Meng Yang ${ }^{2}$, Yanni $\mathbf{L i}^{2}{ }^{2}$, Bin Wu ${ }^{\text {Corresp., }}{ }^{2}$, Xianming Lin ${ }^{\text {Corresp. } 1}$ \\ ${ }^{1}$ Institute of Chinese Medicinal Materials, Hubei Academy of Agricultural Sciences, Enshi, China \\ 2 Chinese Academy of Medical Sciences and Peking Union Medical College, Institute of Medicinal Plant Development, beijing, China \\ Corresponding Authors: Bin Wu, Xianming Lin \\ Email address: bwu@implad.ac.cn, Ixm31@sina.com
}

Pueraria lobata (willd.)Ohwi is a consumable selenium-enriched plant used for medicinal purposes. The molecular response to selenium (Se) stimuli in $P$. lobata is currently unknown. We used RNA-Seq to identify potential genes involved in selenite metabolism and analyzed their expression profiles. We obtained a total of 150,567 unigenes, of which 90,961 were annotated, including 16 structural genes, 14 sulfate transporters, and 13 phosphate transporters thatmay be involved in Se metabolism, and 33 candidate structural genes involved in isoflavone biosynthesis. The genes with a |foldchange| $>2$ and $q$ value $<0.05$ after sodium selenite treatment were identified as differentially expressed genes(DEGs). We obtained a total of 4,246 DEGs, which were enriched in GO terms that included "responseto stimulus", " response to stress", "signal transduction", "response to abiotic stimulus", and "response tochemical". Of the 4,246 DEGs, 1 sulfate transporter and 5 phosphate transporter genes involved Semetabolism, and 9 structural genes involved in isoflavone biosynthesis were up-regulated. The expression patterns of 10 DEGs were selected randomly and validated using qRT-PCR . The Pearson Correlation Coefficient ( $r$ ) was 0.86 , indicating the reliability of RNA-Seq results . 22 Reactive Oxygen Species (ROS) scavenging DEGs were found, 11 of which were up-regulated. 436,624 transcription factors (TFs) correlate $d$ with structural genes were identified that may be involved in Se and isoflavone biosynthesis, respectively, using $r(r>0.7$ or $r<-0.7) .556$ TFs were related to at least one sulfate and phosphate transporter . Our results provided a comprehensive description of gene expression and regulation in response to Se stimuli in P. lobata ., 
1 Transcriptome sequencing and analysis reveals the molecular response to selenium stimuli

\section{2 in Pueraria lobata (willd.) Ohwi}

3 Kunyuan $\mathrm{Guo}^{1}$, Yiwei $\mathrm{Yao}^{2}$, Meng Yang${ }^{2}$, Yanni $\mathrm{Li}^{2}$, Bin $\mathrm{Wu}^{2}$, *, Xianming Lin ${ }^{1}$ *

$4{ }^{1}$ Institute of Chinese Medicinal Materials, Hubei Academy of Agricultural Sciences, Enshi, 5 China

$6{ }^{2}$ Institute of Medicinal Plant Development, Chinese Academy of Medical Sciences and Peking 7 Union Medical College, Beijing, China

8

$9{ }^{*}$ Corresponding authors: Email: bwu@implad.ac.cn (BW); 1xm@sina.com (XL) 


\section{ABSTRACT}

Pueraria lobata (willd.) Ohwi is a consumable selenium-enriched plant used for medicinal purposes. The molecular response to selenium (Se) stimuli in P. lobata is currently unknown. We used RNA-Seq to identify potential genes involved in selenite metabolism and analyzed their expression profiles. We obtained a total of 150,567 unigenes, of which 90,961 were annotated, including 16 structural genes, 14 sulfate transporters, and 13 phosphate transporters that may be involved in Se metabolism, and 33 candidate structural genes involved in isoflavone biosynthesis. The genes with a $\mid$ foldchange $\mid>2$ and $\mathrm{q}$ value $<0.05$ after sodium selenite treatment were identified as differentially expressed genes (DEGs). We obtained a total of 4,246 DEGs, which were enriched in GO terms that included "response to stimulus", "response to stress", "signal transduction", "response to abiotic stimulus", and "response to chemical". Of the 4,246 DEGs, 1 sulfate transporter and 5 phosphate transporter genes involved Se metabolism, and 9 structural genes involved in isoflavone biosynthesis were up-regulated. The expression patterns of 10 DEGs were selected randomly and validated using qRT-PCR. The Pearson Correlation Coefficient (r) was 0.86, indicating the reliability of RNA-Seq results. 22 Reactive Oxygen Species (ROS) scavenging DEGs were found, 11 of which were up-regulated. 436, 624 transcription factors (TFs) correlated with structural genes were identified that may be involved in Se and isoflavone biosynthesis, respectively, using $\mathrm{r}(\mathrm{r}>0.7$ or $\mathrm{r}<-0.7)$. 556 TFs were related to at least one sulfate and phosphate transporter. Our results provided a comprehensive description of gene expression and regulation in response to Se stimuli in P. lobata. 


\section{INTRODUCTION}

Pueraria lobata (Wild.) Ohwi, known as kudzu, is mostly found throughout East Asian countries (He et al., 2019). The dried roots of $P$. lobata have been used medicinally for centuries to treat influenza, muscle stiffness, and other ailments (The Chinese Pharmacopoeia, 2005) with its usage first recorded in the Shengnong Bencao Jing, the first comprehensive work on Chinese Medicine. Isolflavonoids, specifically puerarin and daidzein, are the main bioactive compounds found in the roots of P. lobata (Du et al., 2010). Modern pharmacological studies have revealed important functions of the $P$. lobata root. Puerarin is known to protect the cardiovascular system and may prevent osteoporosis, liver injury, and inflammation (Han et al., 2004) and daidzein decreases blood alcohol levels (Lowe et al., 2008). A series of medicine, and health and food products have been developed using $P$. lobata as the functional ingredient because of its efficacy (http://samr.cfda.gov.cn/WS01/CL0001/).

Selenium ( $\mathrm{Se}$ ) is a trace element essential for humans and animals and is an active ingredient of glutathione peroxidase, which is an antioxidant and has anti-aging and immunity benefits in humans (Fairweather-Tait et al., 2011). A severe selenium deficiency can lead to Keshan disease and Kashin-Beck disease while an overdose can cause hair loss, headache, fatigue and discoloration of the nails (Fairweather-Tait et al., 2011). Plants absorb selenite- and selenate-Se from the soil and transform them into organic Se (Winkel et al., 2015), which is the form predominantly consumed by humans. Current research has only speculated on the transport and transformational processes of selenate and selenite in higher plant species (Winkel et al., 2015). This process can be roughly classified into two stages: selenocysteine (SeCys) formation and Se methylation. In the SeCys formation stage, selenate may be taken up via sulfate transporters (Terry et al., 2000) and selenite may be taken up via phosphate transporters (Barrow et al., 2010; Terry et al., 2000) or Si-transporters (Zhao et al., 2010). Selenite may be further reduced to selenide by sulfite reductase or by glutathione disulfide reductase and glutathione oxidoreductase. Selenide may then be transformed to selenocysteine (SeCys) by cysteine synthase, or by selenide water dikinase, Se-cysteine-tRNA synthase, and cysteine-tRNA 
63 ligase. SeCys may be incorporated into Se-proteins (Aldwin et al., 2012; Hurst et al., 2013), 64 functioning as an antioxidant. SeCys may be transformed into dimethyl diselenide (DMDSe) or 65 selenomethionine (SeMet) during the Se methylation stage and eventually into dimethyl selenide 66 (DMSe) via the methionine cycle by a series of enzymes. DMDSe and DMSe can be emitted to 67 the atmosphere to alleviate the Se toxification of the plant (Winkel et al., 2015).

Few studies have been performed on the molecular mechanism responsible for selenite stimuli in plants. One case study identified 14 selenite-responsive genes in Astragalus racemosus (Hung et al., 2012). Other research has shown that the antioxidative system was activated and photosynthesis and primary metabolism were enhanced under low selenite stress. High selenium stress has been shown to inhibit photosynthesis, primary metabolism, protein ubiquitination, and phosphorylation (Wang et al., 2012).

P. lobata is a selenium-enriched plant primarily composed of selenoproteins and selenopolysaccharides in which organic Se accounted for $82.42 \%$ of the total Se (Du et al., 2010). However, the molecular mechanism responsible for the Se stimuli response in P. lobata is unknown. RNA-seq was used to identify structural genes that may be involved in the biosynthesis of Se and isoflavonoids. We found that selenite may be taken up via phosphate transporters in P. lobata. Transcription factors (TFs) were found to potentially regulate the structural genes in Se and isoflavonoid biosynthesis. Our results are foundational for engineering breeding cultivars of $P$. lobata with high Se compounds.

\section{MATERIALS AND METHODS}

\section{Plant growth conditions and sodium selenite treatment}

Mature stems were cut from one year-old P. lobata cv. GuiYege NO. 1 plants from Huazhong Medicinal Botanical Garden of the Institute of Chinese Medicinal Materials, Hubei Academy of Agricultural Sciences (Enshi, China). The stems were transplanted into sterilized vermiculite in plastic pots $(16 \mathrm{~cm} \times 16 \mathrm{~cm})$ and grown in a greenhouse under a light/dark period of $16 \mathrm{~h} / 8 \mathrm{~h}$ at $25^{\circ} \mathrm{C}$ to control soil moisture and temperature. $P$. lobata seedlings with five leaves were treated with $200 \mathrm{~mL}$ sodium selenite at $0,1,5,15,25,35,55 \mathrm{mg} / \mathrm{L}$, respectively, using an 
90

91

92

93

94

95

even spray on the vermiculite in the pots. The control was treated with distilled water. Every treatment was replicated three times.

\section{Measurement of physiological and biochemical indexes}

The root length was measured on the 9th day after the sodium selenite treatment using a vernier caliper. Biochemical indexes were measured on the day of treatment and then then the 1st, 3th, 5th, 7th and 9th days of treatment thereafter. The total selenium content was measured using hydride atomic fluorescence spectrometry (Fernanda et al., 2016). The activity of superoxide dismutase (SOD) was determined as described by Zhang et al (2012b) and the content of malondialdehyde (MDA) was assayed using the protocol from a previous study (Feng et al., 2009b). The correlation analyses of the differences between sampling days and concentrations of SOD and MDA were performed by SPSS 19 (Qiu et al., 2017).

\section{RNA extraction, library construction, and sequencing}

Samples were collected on the day of treatment and the 1st, 3th, 5th, 7th and 9th days following the treatment with $25 \mathrm{mg} / \mathrm{L}$ sodium selenite. The roots were washed with distilled water, blotted with dry filter paper, and immediately frozen in liquid nitrogen. Total RNA was extracted, treated, and measured following the protocol from our previous study (Wu et al. 2015). Total RNA was extracted using the TRizol reagent according to the manufacturer's instructions and was digested to eliminate the residual genomic DNA using RNase-free DNase. The Agilent Technologies 2100 Bioanalyzer was used to measure and quantify the total RNA.

RNA-Seq libraries were constructed and sequenced as follows: the mRNA of each sample was separated from the total RNA using oligo (dT) magnetic beads; the mRNA were then cleaved into short fragments; the short fragments were used as templates and the first-strand cDNA was synthesized by reverse transcriptase and random primers. The RNA templates were then removed and the second-strand cDNA was synthesized using dNTPs, DNA polymerase I, and RNase $\mathrm{H}$. These short, double-cDNA fragments were purified with DNA clean beads. The short cDNA fragments were ligated with the Illumina paired-end adaptors and purified with DNA clean beads following end reparation and A-tailing. PCR was used to selectively enrich 
117

118

119

120

121

122

123

124

125

126

127

128

129

130

131

132

133

134

135

136

137

138

139

140

141

142

143

144

145

DNA fragments with adapter molecules on both ends and to create the final cDNA library. The quality of the cDNA library was measured using the Agilent 2100 Bioanalyzer. The libraries were then sequenced from the 5' and 3' ends using the Illumina sequencing system.

\section{De novo transcriptome assembly and annotation}

RNA-Seq reads were de novo assembled using Trinity to obtain high-quality transcription sequences with the default parameters (Grabherr et al., 2011). The assembly unigenes were compared with sequences in the CDD database using BLAST (Marchler et al., 2013), KOG (Koonin et al., 2004), COG (Tatusov et al., 2000), NR and NT (https://blast.ncbi.nlm.nih.gov/Blast.cgi), PFAM (Finn et al., 2016), Swissprot (Boeckmann et al., 2005), TrEMBL (Boeckmann et al., 2003), GO (Ashburner et al., 2000) and KEGG (Kanehisa et al., 2004) for annotations with E-value $10^{-5}$ as the cutoff.

\section{Illumina data processing and gene expression profiles quantification}

Raw reads were processed using in-house Perl scripts in FASTQ format. Clean reads were obtained after removing low-quality reads and those containing adapters and poly-N using Trimmomatic (Bolger et al., 2014). Gene expression levels were determined by TPM

(Transcripts Per Kilobase of exon model per Million mapped read) to calculate the fold change. The read count was calculated using Salmon (Patro et al., 2017) and was loaded into DESeq2 (Anders \& Huber, 2010) to calculate the P value for differential expression analysis. Genes that had a $\mid$ foldchange $\mid \geq 2$ and $\mathrm{q}$ value $<0.05$ identified by DESeq were defined as differentially expressed genes (DEGs).

\section{TFs identification}

Sequences of the unigenes were compared to the sequences in the iTAK database to analyze TFs in P. lobata using the default parameters (Zheng et al., 2016).

\section{Validation of differential expression genes by qRT-PCR}

qRT-PCR were performed as described by our previous paper (Wu et al.,2015). 10 randomly selected genes were validated. Gene-specific primers were designed by Primer5 and the primer sequences are listed in Table S1. The 40S ribosomal protein S8 was used as an internal control (He et al., 2019). The Pearson Correlation Coefficient (r) was calculated between the mean results of qRT-PCR and RNA-Seq. 
146

147

148

149

150

151

152

153

154

155

156

157

158

159

160

161

162

163

164

165

166

167

168

169

170

171

172

173

\section{RESULTS}

\section{Physiological and biochemical index of $P$. lobata after sodium selenite treatment}

The total Se content in P. lobata seedlings showed an increase and followed by a decrease with an increased sodium selenite concentration; plant leaves accumulated more Se than the stems. The total Se content in the seedling was lower when the concentration of sodium selenite was less than $5 \mathrm{mg} / \mathrm{L}$, however the total Se content was maximized when the concentration was $25 \mathrm{mg} / \mathrm{L}$. The accumulation of selenium in $P$. lobata seedlings began to decline when the concentration was more than $35 \mathrm{mg} / \mathrm{L}$ (Figure S1). The root length tended to be the same under the Se accumulation and the sodium selenite treatments (Figure S2). SOD content showed an initial increase and then decrease. SOD reached its maximum content at $25 \mathrm{mg} / \mathrm{L}, 5$ days after treatment, and then decreased gradually (Figure 1A). The content of MDA tended to increase with the sodium selenite concentration and treatment time (Figure 1B). Correlation analyses were conducted on the differences between sampling days and concentrations of SOD. MDA was also conducted. Results for SOD activity revealed that the treatment concentration of sodium selenite was negatively correlated on the 7th day and significantly negatively correlated on the 9th day (Table S2). The treatment from the 1st day was positively correlated with those of the 3rd, 5th, and 7th days. The treatment from the 3rd day was significantly positively correlated with that of the 5 th day. The treatment from the 5 th day was positively correlated with that of the 7th day. The treatment from the 7th day was significantly positively correlated with that of the 9th day. Results showed that the treatment concentration of sodium selenite was positively correlated the MDA content from the 1st day of treatment and was significantly positively correlated with the treatments from the 5th, 7th and 9th days, respectively (Table S3). There was a significant positive correlation between the treatment from the 1 st day and those of the $3 \mathrm{rd}$, 5th, 7th and 9th days, respectively.

\section{RNA-Seq sequencing and assembly}

The 18 mRNA samples from days $0,1,3,5,7,9$ after sodium selenite treatment were sequenced on the Illumina platform. We obtained more than $6 \mathrm{~Gb}$ of data from every sample for the downstream analysis. The transcriptome data was assembled and 150,567 unigenes were 
174

175

176

177

178

179

180

181

182

183

184

185

186

187

188

189

190

191

192

193

194

195

196

197

198

199

200

obtained using Trinity (Grabherr et al., 2011). The N50 length and average read length of the assembly sequence were $1,742 \mathrm{bp}$ and $962.46 \mathrm{bp}$, respectively. The length distribution and GC content of the unigenes was calculated (Figure S3).

\section{Gene function annotation and categorization}

The assembled 150,567 unigenes were compared with sequences found in the CDD, KOG, $\mathrm{Nr}$, Nt, Pfam, Swissport, TrEMBL, GO, and KEGG by BLAST (version 2.2.26) databases using default parameters. There were 46, 439, 33, 401, 59,266, 69,142, 31,796, 58,101, 58,917, 64,796, and 5,415 resulting annotations found (Table S4). 90,961 total unigenes were annotated.

$\mathrm{GO}$ ontology describes the molecular function, cellular component, and biological processes of genes. 67 total secondary classifications were obtained and the top five enriched GO terms were cell (GO: 0005623), cell part (GO: 0044464), cellular process (GO: 0009987), binding (GO: 0005488), and metabolic process (GO: 0008152) (Figure 2).

Identification of structural genes potentially involved in the biosynthesis of selenium and isoflavones

P. lobata is an important Se-enriched plant. However, its Se-related structure is unknown. The KEGG database was used to annotate and assign genes with Ko terms to specific metabolic pathways. 5,415 annotated genes in KEGG were further classified into four categories: genetic information processing, environmental information processing, metabolism, and cellular processes. The top five subcategories were signal transduction, carbohydrate metabolism, folding sorting and degradation, and overview (Figure 3). A total of of 16 Se-related structural genes, 14 sulfate transporters, and 13 phosphate transporters were identified when KEGG was combined with $\mathrm{Nr}$ annotation (Table S5).

The isoflavone-related genes were further mined to determine whether the biosynthesis of the isoflavone in P. lobata was affected by sodium selenite treatment. The biosynthesis of isoflavones were divided into three stages: the phenylpropane pathway, the flavonoids pathway, and the isoflavone pathway (Han et al., 2015; He et al., 2011; Li et al., 2016a; Li et al., 2016b; Wang et al., 2016; Wang et al., 2017). Five PALs, three $C 4 H$ s, and five $4 C L$ s were found in the 
201 phenylpropane pathway; three $C H S$ s and three $C H I$ s were found in the flavonoid pathway; one

202

203

204

205

206

207

208

209

210

211

212

213

214

215

216

217

218

219

220

221

222

223

224

225

226

227

IFS, seven HIDs, one HIAOMT, two I7OMTs, and three IF7MATs were found in the isoflavone pathway. A total of 32 genes potentially involved in isoflavone biosynthesis in $P$. lobata were identified in this study (Figure S4, Table S6).

\section{Comprehensive analysis of DEGs}

Genes with a $\mid$ foldchange $\mid>2$ and $\mathrm{q}$ value $<0.05$ after sodium selenite treatments for different lengths of time were identified as differentially expressed genes (DEGs). A total of 4,246 DEGs were obtained (Table S7). 10 DEGs were selected randomly for analysis by qRTPCR (Figures 4A-J) to validate the reliability of the RNA-Seq results (Table S8). The results between RNA-Seq and qRT-PCR were analyzed. The Pearson Correlation Coefficient (r) is approximately 0.86 (Figure 4K), which indicates that the results of RNA-Seq and qRT-PCR are positively linearly related. The expression levels in RNA-Seq reflect the differences in gene expression after selenium treatment.

1,538 DEGs were identified after the first day of selenite treatment, including 465 upregulated genes and 1,073 down-regulated genes. 1,386 DEGs were identified on the third day of treatment, including 559 up-regulated genes and 827 down-regulated genes. 1,348 DEGs were identified on the fifth day of treatment, including 407 up-regulated genes and 941 downregulated genes. 2,510 DEGs were identified on the seventh day of treatment, including 883 upregulated genes and 1,627 down-regulated genes. 1,556 DEGs were identified on the ninth day of treatment, including 1,032 up-regulated genes and 524 down-regulated genes (Table 1).

We focused on the functional enrichment of the 4246 DEGs. GO enrichment showed that "response to stimulus", "response to stress", "signal transduction", "response to abiotic stimulus", and "response to chemical" were the top five classifications (Figure 5), which indicated that sodium selenite was a stress molecule. KEGG enrichment indicated that sodium selenite affected plant hormone signal transduction, MAPK signaling pathway-plant, starch and sucrose metabolism, ribosome biogenesis in eukaryotes, flavonoid biosynthesis, and isoflavonoid biosynthesis (Figure S5). 
228

229

230

231

232

233

234

235

236

237

238

239

240

241

242

243

244

245

246

247

248

249

250

251

252

253

254

Cluster analysis was performed on gene expression profiles of RNA-seq libraries from 18 samples according to Ernst's STEM algorithms arithmetic to assess the dynamic changes of DEGs after sodium selenite treatment (Ernst et al., 2006). Four representative expression patterns were obtained (Figure 6) and classified as subclusters. 1,443 genes were down-regulated as a whole after selenium treatment in subcluster 1; these were mainly involved in DNA metabolism, material transport, synthesis of primary metabolites, and stress-related processes. 881 genes in subcluster 2 were down-regulated on the first day and then up-regulated at another time, and were mainly involved in disease resistance, stress tolerance, material transport and cytoskeleton construction. 505 genes in subcluster 3 were up-regulated as a whole after selenium treatment; these were mainly involved in the secondary metabolism synthesis, response to toxin metabolism, and response to stress. 297 genes were up-regulated and then down-regulated on the first day in subcluster 4, and were mainly involved in regulating material transport, DNA repair, and resistance reactions.

\section{DEGs overlap at five different time points}

DEGs were analyzed continuously after sodium selenite treatment. When compared with the control (Figure 7), 160 DEGs were observed, including 101 up-regulated DEGs and 59 down-regulated DEGs (Table S9). Among the up-regulated DEGs, 9 genes (T_39191_c1_g1, T_39355_c1_g4, T_41331_c0_g6, T_42572_c0_g2, T_44141_c2_g4, T_44939_c1_g2, T_44939_c1_g3, T_44939_c1_g5, T_45070_c2_g1) are potentially involved in the biosynthesis of isoflavones; 1 gene (T_40246_c1_g1) encoded the selenium binding protein, one gene (T_42307_c2_g3) encoded glutathione s-type transferase and two genes (T_44116_c2_g1, T_44116_c2_g3) encoded phosphate transporters. Among those down-regulated genes, two MYB transcription factors, MYB39 (T_36389_c0_g1) and R2R3-MYB (T_41871_c2_g5), one thioredox protein (T_44132_c2_g1), and other genes that encoded TMV disease-resistant proteins and ion transport proteins were found.

\section{Reactive Oxygen Species (ROS) scavenging genes in $P$. lobata}

Low selenium stress has been shown to activate the antioxidant system in plants to scavenge 
255

256

257

258

259

260

261

262

263

264

265

266

267

268

269

270

271

272

273

274

275

276

277

278

279

280

281

ROS and enhance its ability to withstand a variety of oxidative stresses (Wang et al., 2012). ROS scavenging genes were mined from $P$. lobata, resulting in the up-regulation of 11 SODs, 4

Catalase $(C A T \mathrm{~s}), 4$ Ascorbate peroxidase $(A P X \mathrm{~s}), 11$ Glutathione peroxidase $(G P X \mathrm{~s}), 55$

Glutathione-S- Transferase (GSTs), and 5 Monodehydroascorbate reductase (MDHARs), in which $1 S O D, 1 A P X$, and 9 GSTs were identified (Table S10).

\section{Transcription factor analysis}

A total of 2,192 transcription factors (TFs) were identified by iTAK software, which were classified into 54 families (Table S11). Among the 2,192 TFs, 241 TFs representing 39 families were differentially expressed after selenium treatment. In order to clarify the regulatory role of TFs in selenium treatment in P. lobata co-expression analysis was performed with TFs among 75 genes, including 16 structural genes, 14 sulfate transporters, and 13 phosphate transporters genes related to selenium metabolism, and 32 structural genes potentially involved in isoflavone biosynthesis of $P$. lobata. $436 \mathrm{TF}$ were found to be related to at least one structural gene in selenium metabolism using the Pearson correlation coefficient $r(r>0.7$ or $r<-0.7)$ as the threshold (Table S12). 556 TFs were related to at least one of the sulfate transporters and phosphate transporters (Table S13). 624 TFs were related to at least one isoflavone-related gene (Table S14).

\section{DISCUSSION}

P. lobata is a Se-enriched plant with an important role in Chinese herbal medicine (Du et al., 2010). However, the molecular mechanism responsible for its response to sodium selenite stimuli is unknown. In our study, the content of selenium in $P$. lobata under different concentrations of sodium selenite was investigated and revealed a pattern of an increase followed by a decrease in selenium content. A maximum selenium content was achieved at a concentration of $25 \mathrm{mg} / \mathrm{L}$ of sodium selenite. The selenium content in leaves was higher than in the stems, which was consistent with results from other studies (Han et al., 2013; Han et al., 2015). We measured the activity of SOD in P. lobata treated with different concentrations of sodium selenite. The results showed that an increase of treatment time did not significantly change the activity of SOD in the 
282

283

284

285

286

287

288

289

290

291

292

293

294

295

296

297

298

299

300

301

302

303

304

305

306

307

308

control group. However, an increased concentration of sodium selenite caused an increase in the activity of SOD in P. lobata followed by a decrease. The trend was most obvious on the fifth day of treatment when the concentration was $25 \mathrm{mg} / \mathrm{L}$. MDA content was determined and the results showed that MDA in P. lobata decreased and then increased with longer treatment times and greater concentrations. Our results indicate that the activity of SOD and the content of MDA in P. lobata could be increased using a treatment of low concentration sodium selenite. These results were consistent with results from previous studies (Han et al., 2013; Han et al., 2015). The root length, MDA content, and SOD activity under different concentrations of sodium selenite further supported $25 \mathrm{mg} / \mathrm{L}$ as a suitable treatment concentration.

The transciptome of $P$. lobata seedlings was sequenced after treatment with $25 \mathrm{mg} / \mathrm{L}$ sodium selenite and the DEGs were identified. 150,567 unigenes were obtained, of which 4,246 were DEGs. The enriched GO terms of DEGs respond to stimulus, stress, signal transduction, abiotic stimulus, and chemicals, which are similar to the responses of sodium selenate in Astragalus chrysochlorus (Özgür et al., 2015). This result is consistent with those of previous studies that the molecular mechanisms of plants that absorb selenite and selenate share some common pathways ( $\mathrm{Li}$ et al., 2008; Yu et al., 2008). Sodium selenite may be taken up by phosphate transporters, because the expression profiles of 5 phosphate transporters genes were up-regulated after applying sodium selenite, and 2 genes (T_44116_c2_g1, T_44116_c2_g3) were continuously up-regulated. Phosphorus is known to be one of the macronutrients required for plant growth and development and a phosphate transporter is needed to absorb phosphate fertilizer. Therefore, we suggest that the absorption of sodium selenite and phosphate fertilizer may compete with one another. The use of these two fertilizers at the same time is contraindicated for the cultivation of Se-enriched plants.

ROS can damage cell membranes and other components and harm plants (Nandini et al., 2016). Low selenium stress has been reported to activate the antioxidant system to scavenge ROS and enhance a plant's ability to withstand a variety of oxidative stresses (Wang et al., 2012). We identified DEGs encoding major ROS scavenging enzymes, including SOD, CAT, APX, 
309

310

311

312

313

314

315

316

317

318

319

320

321

322

323

324

325

326

327

328

329

330

331

332

333

334

335

GPX, GST, and MDHAR (Apel et al., 2004). We found that $1 S O D, 1 A P X$, and 9 GSTs were upregulated when compared with the control, which might enhance the anti-oxidation mechanisms after sodium selenite treatment in P. lobata (Table S7). GST (T_42307_c2_g3) was up-regulated consistently after selenite treatment.

To address the question of whether sodium selenite treatment in $P$. lobate would promote the biosynthesis of isoflavone, we analyzed the expression profiles of isoflavone-related structural genes and found that 9 genes were up-regulated (Table S6), indicating that isoflavone biosynthesis was promoted after $25 \mathrm{mg} / \mathrm{L}$ sodium selenite treatment. These results are consistent with previous studies reporting that the total flavonoid and chlorogenic acid contents were enhanced when the application dose did not exceed $2.0 \mathrm{mg} / \mathrm{kg}$ (Li et al., 2010). These results suggest that the content of Se and other active compounds could be enhanced in medicinal plants by applying a suitable concentration of sodium selenite.

We identified the role of TFs in regulating selenium metabolism for the first time. 436 TFs were related with structural genes in Se metabolism (Table S12) and 556 TFs were co-expressed with sulfate transporters or phosphate transporters (Table S13). Our results provided a comprehensive overview of the gene expression and regulation in response to selenium stimuli in P. lobata.

\section{CONCLUSION}

RNA-Seq was used to identify the molecular mechanism involved in the response to Se stimili in P. lobata. We found 150,567 unigenes, of which 90,961 were annotated. Among these annotated genes, 16 structural genes, 14 sulfate transporters, and 13 phosphate transporters were potentially involved in Se metabolism and 32 structural genes were related to isoflavone biosynthesis. We obtained 4,246 DEGs, of which 1 sulfate transporter and 5 phosphate transporter genes involved in Se metabolism and 9 structural genes involved in isoflavone biosynthesis were up-regulated. Furthermore, twenty-two ROS scavenging DEGs were identified, of which 11 were up-regulated. Finally, we identified some TFs that are potentially involved in Se metabolism and isoflavone biosynthesis. In order to cultivate Se-enriched plants, sodium 
336

337

338

339

340

341

342

343

344

selenite and phosphate fertilizer should not be applied at the same time. The content of Se and active compounds may be enhanced in plants used for medicinal purposes by applying a suitable concentration of sodium selenite.

\section{REFERENCES}

Aldwin Suryo R, Davies M J. 2012. Selenium-containing amino acids as direct and indirect antioxidants. Lubmb Life 64: 863-871 DOI 10.1002/iub.1084.

Anders S, Huber W. 2010. Differential expression analysis for sequence count data. Genome Biology 11: R106 DOI 10.1186/gb-2010-11-10-r106.

Apel K, Hirt H. 2004. Reactive oxygen species: metabolism, oxidative stress, and signal transduction. Annual Review of Plant Biology 55: 373-399 DOI 10.1146/annurev.arplant.55.031903.141701.

Ashburner M, Ball C A, Blake J A, Botstein D, Butler H, Cherry J M, Davis A P, Dolinski K, Dwight S S, Eppig J T, Harris M A, Hill D P, Issel-Tarver L, Kasarskis A, Lewis S, Matese J C, Richardson J E, Ringwald M, Rubin G M, Sherlock G. 2000. Gene ontology: tool for the unification of biology. Nature Genetics DOI 25: 2529. $10.1038 / 75556$.

Barrow N J, Whelan B R. 2010. Testing a mechanistic model. VIII. The effects of time and temperature of incubation on the sorption and subsequent desorption of selenite and selenate by a soil. European Journal of Soil Science 40(1): 29-37 DOI 10.1111/j.1365-2389.1989.tb01251.x.

Boeckmann B, Bairoch A, Apweiler R, Blatter M C, Estreicher A, Gasteiger E, Martin M J, Michoud K, O'Donovan C, Phan I, Pilbout S, Schneider M. 2003. The SWISS-PROT protein knowledgebase and its supplement TrEMBL in 2003. Nucleic Acids Research 31(1): 365-370 DOI 10.1093/nar/gkg095.

Boeckmann B, Blatter M C, Famiglietti L, Hinz U, Lane L, Roechert B, Bairoch A. 2005. Protein variety and functional diversity: Swiss-Prot annotation in its biological context. Comptes Rendus Biologies 328(10): 882-899 DOI 10.1016/j.crvi.2005.06.001.

Bolger A M, Lohse M, Usadel B. 2014. Trimmomatic: a flexible trimmer for Illumina sequence data. Bioinformatics, 30(15):2114-2120 DOI 10.1093/bioinformatics/btu170.

Du G, Zhao H Y, Zhang Q W, Li G H, Yang F Q, Wang Y, Li Y C, Wang Y T. 2010. A rapid method for simultaneous 
determination of 14 phenolic compounds in Radix Puerariae using microwave-assisted extraction and ultra high performance liquid chromatography coupled with diode array detection and time-of-flight mass spectrometry. Journal of Chromatography $A \quad 1217(5): \quad 705-714 \quad$ DOI 10.1016/j.chroma.2009.12.017.

Ernst J, Bar-Joseph Z. 2006. STEM: a tool for the analysis of short time series gene expression data. Bmc Bioinformatics 7: 191 DOI 10.1186/1471-2105-7-191.

Fairweather T S J, Bao Y, Broadley M R, Collings R, Ford D, Hesketh J E, Hurst R. 2011. Selenium in human health and disease. Antioxidants Redox Signaling 14(7): 1337-1383 DOI 10.1089/ars.2010.3275.

Fan L L, O'Keefe D D, Jr P W. 1985. Pharmacologic studies on radix puerariae: effect of puerarin on regional myocardial blood flow and cardiac hemodynamics in dogs with acute myocardial ischemia. Chinese Medical Journal 98(11): 821-832 DOI 10.1016/0021-9150(81)90059-9.

Feng R, Wei C, Tu S, Wu F. 2009b. Effects of Se on the uptake of essential elements in Pteris vittata L. Plant Soil 325(1): 123-132 DOI 10.1007/s11104-009-9961-9.

Fernanda A S, Lindomar A P, Antonio M S, Laura F, Víctor C, Sergio L C F. 2016. Development of a MSFIA system for sequential determination of antimony, arsenic and selenium using hydride generation atomic fluorescence spectrometry. Talanta 156-157: 29-33 DOI 10.1016/j.talanta.2016.04.063.

Finn R D, Coggill P, Eberhardt R Y, Eddy S R, Mistry J, Mitchell A L, Potter S C, Punta M, Qureshi M, Sangradorvegas A. 2016. The Pfam protein families database: towards a more sustainable future. Nucleic Acids Research 44(D1): D279-D285 DOI 10.1093/nar/gkv1344.

Grabherr M G, Haas B J, Yassour M, Levin J Z, Thompson D A, Amit I, Adiconis X, Fan L, Raychowdhury R, Zeng Q D. 2011. Full-length transcriptome assembly from RNA-Seq data without a reference genome. Nature Biotechnology 29(7): 644-652 DOI 10.1038/nbt.1883.

Han D, Li X H, Xiong S L, Tu S X, Chen Z G, Li J P, Xie Z J. 2013. Selenium uptake, speciation and stressed response of Nicotianatabacum L. Environmental and Experimental Botany, 95(8): 6-14 DOI 10.1016/j.envexpbot.2013.07.001

Han D, Xiong S L, Tu S X, Liu J C, Chen C. 2015. Interactive effects of selenium and arsenic on growth, antioxidant system, arsenic and selenium species of Nicotianatabacum L. Environmental and 
390

391

392

393

394

395

396

397

398

399

400

401

402

403

404

405

406

407

408

409

410

411

412

413

414

415

416

Experimental Botany, 117: 12-19 DOI 10.1016/j.envexpbot.2015.04.008.

Han R, Takahashi H, Nakamura M, Yoshimoto N, Suzuki H, Shibata D, Yamazaki M, Saito K. 2015. Transcriptomic landscape of Pueraria lobata demonstrates potential for phytochemical study. Front Plant Science 6: 426 DOI 10.3389/fpls.2015.00426

He M, Yao Y, Li Y, Yang M, Wu B, Yu D. 2019. Comprehensive transcriptome analysis reveals genes potentially involved in isoflavone biosynthesis in Pueraria thomsonii Benth. Plos One 14(6): e0217593 DOI 10.1371/journal.pone.0217593.

He X Z, Blount J W, Ge S, Tang Y, Dixon R A. 2011. A genomic approach to isoflavone biosynthesis in kudzu (Pueraria lobata). Planta 233(4): 843-855 DOI 10.1007/s00425-010-1344-1.

Hung C Y, Holliday B M, Kaur H, Yadav R, Kittur F S, Xie J. 2012. Identification and characterization of selenateand selenite-responsive genes in a Se-hyperaccumulatorAstragalus racemosus. Molecular Biology Reports 39(7): 7635-7646 DOI 10.1007/s11033-012-1598-8.

Hurst R, Collings R, Harvey L J, King M, Hooper L, Bouwman J, Gurinovic M, Fairweather-Tait S J. 2013. EURRECA-Estimating Selenium Requirements for Deriving Dietary Reference Values. C R C Critical Reviews in Food Technology 53(10): 1077-1096 DOI 10.1080/10408398.2012.742861.

Kanehisa M, Goto S, Kawashima S, Okuno Y, Hattori M. 2004. The KEGG resource for deciphering the genome. Nucleic Acids Research 32: D277-280 DOI 10.1093/nar/gkh063.

Koonin E V, Fedorova N D, Jackson J D, Jacobs A R, Krylov D M, Makarova K S, Mazumder R, Mekhedov S L, Nikolskaya A N, Rao B S. 2004. A comprehensive evolutionary classification of proteins encoded in complete eukaryotic genomes. Genome Biology 5: R7-R7 DOI 10.1186/gb-2004-5-2-r7.

Li H F, McGrath S P, Zhao F J. 2008. Selenium uptake, translocation and speciation in wheat supplied with selenate or selenite. New Phytologist 178: 92-102 DOI 10.1111/j.1469-8137.2007.02343.x

Li J, Li C F, Gou J B, Wang X, Fan R Y, Zhang Y S. 2016a. An Alternative Pathway for Formononetin Biosynthesis in Pueraria lobata. Frontiers in Plant Science 7: 861 DOI 10.3389/fpls.2016.00861.

Li J, Li C F, Gou J B, Zhang Y S. 2016b. Molecular Cloning and Functional Characterization of a Novel Isoflavone 3'-O-methyltransferase from Pueraria lobata. Frontiers in Plant Science 7: 793 DOI 10.3389/fpls.2016.00793.

Peer] reviewing PDF | (2019:09:41362:2:0:NEW 3 Feb 2020) 
417

418

419

420

421

422

423

424

425

426

427

428

429

430

431

432

433

434

435

436

437

438

439

440

441

442

443

444

Li Y M, Sun Y X, Liu D H. 2010. Effects of Applying Selenium on Contents of Total Flavonoid, Chlorogenic Acid and Selenium in Flower of Chrysanthemum morifolium. Soil 42(4): 618-623 DOI 10.13758/j.cnki.tr.2010.04.025.

Lowe E D, Gao G Y, Johnson L N, Keung W M. 2008. Structure of daidzin, a naturally occurring anti-alcoholaddiction agent, in complex with human mitochondrial aldehyde dehydrogenase. Journal of Medicinal Chemistry 51(15):4482-7 DOI 10.1021/jm800488j.

Marchler B A, Zheng C, Chitsaz F, Derbyshire M K, Geer L Y, Geer R C, Gonzales N R, Gwadz M, Hurwitz D I, Lanczycki C J. 2013. CDD: conserved domains and protein three-dimensional structure. Nucleic Acids Research 41: 348-352 DOI 10.1093/nar/gks1243.

Nandini Y, Samir S. 2016. Reactive Oxygen Species, Oxidative stress and ROS scavenging system in plants. Journal of Chemical and Pharmaceutical Research 8: 595-604 DOI 10.1371/journal.pone.0217593.

Özgür Ç, Neslihan T K, Arı Ş, Baohong Z. 2015. De Novo Transcriptome Assembly and Comparative Analysis Elucidate Complicated Mechanism Regulating Astragalus chrysochlorus Response to Selenium Stimuli. Plos One 10(10): e0135677 DOI 10.1371/journal.pone.0135677.

Patro R, Duggal G, Love M I, Irizarry R A, Kingsford C. 2017. Salmon provides fast and bias-aware quantification of transcript expression. Nature Methods, 14(4):417-419 DIO 10.1038/nmeth.4197.

Qiu Z X, Sha Z S, Che X M, Wang M Y. 2017. Correlation analysis of ADAMTS-4, VCAM-1, and TAK1 expression in cartilage tissue from spine tuberculosis. Genetics and Molecular Research 16(3): gmr16038961 DIO 10.4238/gmr16038961.

Tatusov R L, Galperin M Y, Natale D A, Koonin E V. 2000. The COG database: a tool for genome-scale analysis of protein functions and evolution. Nucleic Acids Research 28(1): 33-36 DOI 10.1093/nar/28.1.33.

Terry N, de Souza M P, Am T A Z. 2000. Selenium in higher plants [Review]. Annual Review of Plant Physiology \& Plant Molecular Biology 51: 401-432 DOI 10.1146/annurev.arplant.51.1.401.

The Chinese Pharmacopoeia. The Chemical Industry Publishing House, Beijing, China 2005: 203.

Wang X, Fan R, Li J, Li C, Zhang Y. 2016. Molecular Cloning and Functional Characterization of a Novel (Iso)flavone 4',7-0-diglucoside Glucosyltransferase from Pueraria lobata. Front Plant Science 7: 387 DOI 10.3389/fpls.2016.00387.

Wang X, Li C, Zhou C, Li J, Zhang Y. 2017. Molecular characterization of the C-glucosylation for pueraria

Peer] reviewing PDF | (2019:09:41362:2:0:NEW 3 Feb 2020) 
biosynthesis in Pueraria lobata. Plant Journal 90(3): 535-546 DOI 10.3389/fpls.2016.00387.

446

447

448

449

450

451

452

453

454

455

456

457

458

459

460

461

462

463

464

465

466
Wang Y D, Wang X, Wong Y S. 2012. Proteomics analysis reveals multiple regulatory mechanisms in response to selenium in rice. Journal of Proteomics 75(6): 1849-1866 DOI 10.1016/j.jprot.2011.12.030.

Winkel L H E, Vriens B, Jones G D, Schneider L S, Bañuelos G S. 2015. Selenium Cycling Across Soil-PlantAtmosphere Interfaces: A Critical Review. Nutrients 7(6): 4199-4239 DOI 10.3390/nu7064199.

Wu B, Long Q, Gao Y, Wang Z, Shao T, Liu Y, Li Y, Ding W. 2015. Comprehensive characterization of a time-461 course transcriptional response induced by autotoxins in Panax ginseng using RNA-Seq. BMC Genomics 462(16): 1010 DOI 10.1186/s12864-015-2151-7.

Yu X Z, Gu J D. 2008. Differences in uptake and translocation of selenate and selenite by the weeping willow and hybrid willow. Environmental Science \& Pollution Research 15(6): 499-508 DOI 10.1007/s11356008-0036-x.

Zhao X Q, Mitani N, Yamaji N, Shen R F, Ma J F. 2010. Involvement of silicon influx transporter OsNIP2;1 in selenite uptake in rice. Plant Physiology 153(4): 1871-1877 DOI 10.1104/pp.110.157867.

Zhang M, Hu C, Zhao X, Tan Q, Sun X, Cao A, Cui M, Zhang Y. 2012b. Molybdenum improves antioxidant and osmotic-adjustment ability against salt stress in Chinese cabbage (Brassica campestris L. ssp. Pekinensis). Plant Soil 355(1): 375-383 DOI 10.1007/s11104-011-1109-z.

Zheng Y, Jiao C, Sun H, Rosli H G, Pombo M A, Zhang P, Banf M. 2016. iTAK: A Program for Genome-wide Prediction and Classification of Plant Transcription Factors,Transcriptional Regulators, and Protein Kinases. Molecular Plant 9(12): 1667-1670 DOI 10.1016/j.molp.2016.09.014. 


\section{Table $\mathbf{1}$ (on next page)}

The number of up- and down-regulated DEGs based comparison with 0 day As control (|foldchange $>2$; q value $<0.05$ )

D0, D1, D3, D5, D7, D9 represent different day after sodium selenite treatment. 
1 Table 1. The number of up- and down-regulated DEGs based comparison with 0 day

2 As control (|foldchange $\mid>2$; q value $<0.05$ )

\begin{tabular}{cccc}
\hline Comparison & Up-regulated & Down-regulated & Total \\
\hline D1 vs D0 & 465 & 1073 & 1538 \\
D3 vs D0 & 559 & 827 & 1,386 \\
D5 vs D0 & 407 & 941 & 1,348 \\
D7 vs D0 & 883 & 1,627 & 2,510 \\
D9 vs D0 & 1,032 & 524 & 1,556 \\
\hline
\end{tabular}


Figure 1

\section{Measurement of SOD and MDA}

A

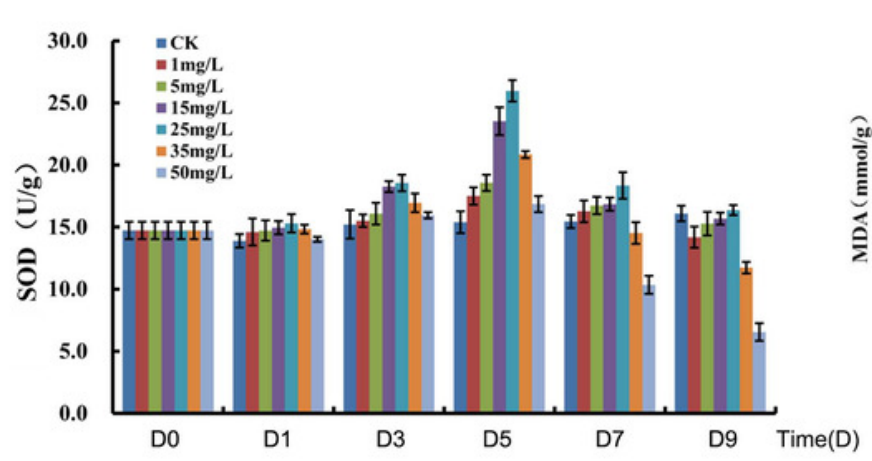

B

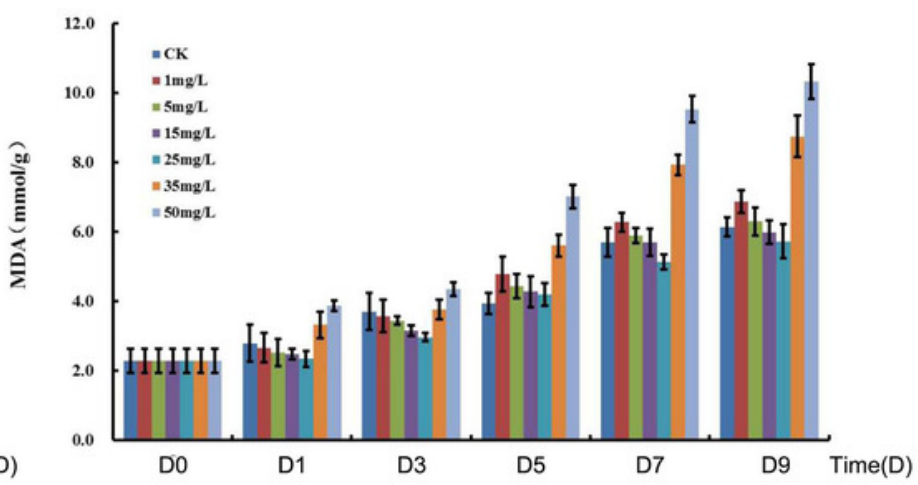




\section{Figure 2}

GO categories of P.lobata unigenes

Percent of unigenes assigned to partial subcategories of the cellular component (blue), molecular function (red) and biological process (green) are represented.

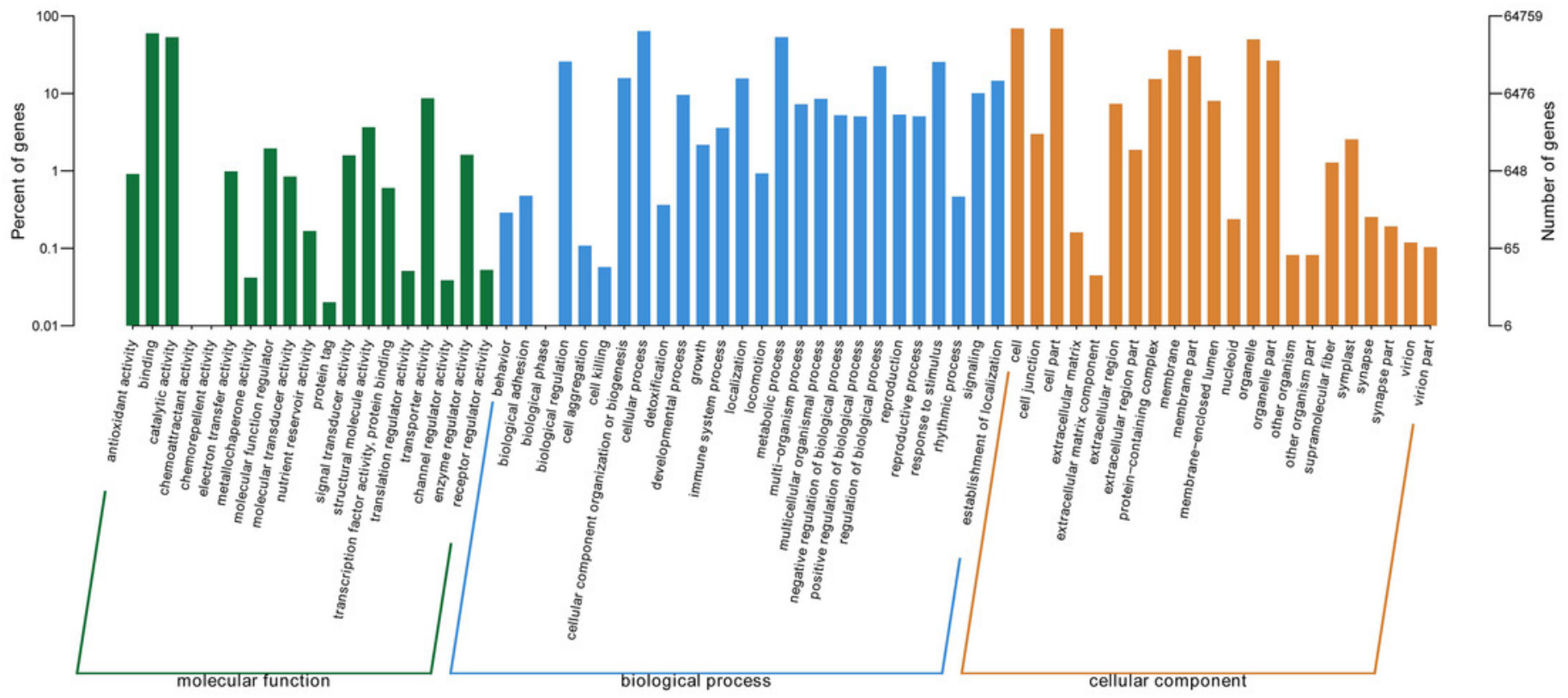


Figure 3

KEGG categories of P.lobata unigenes

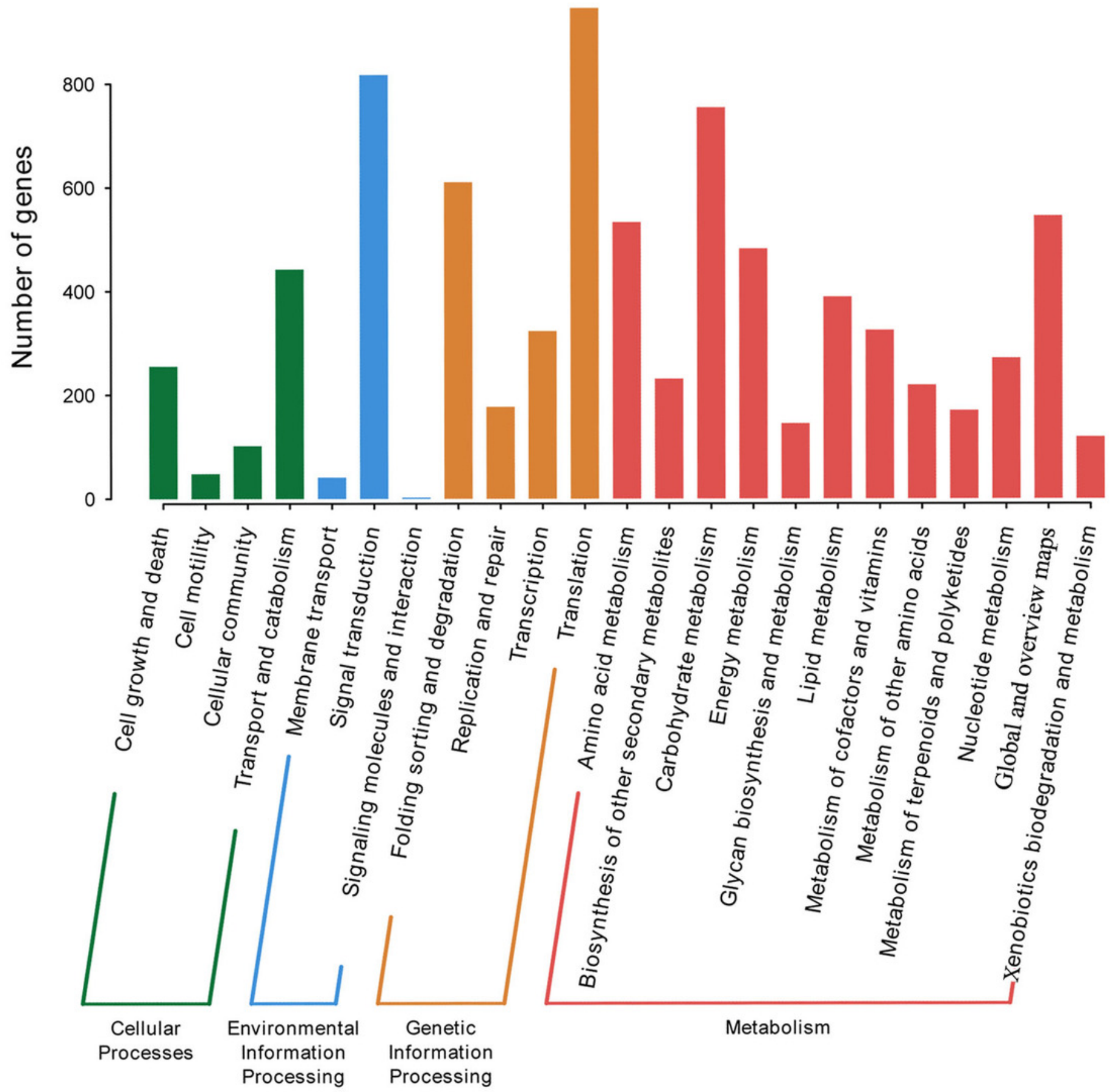


Figure 4

Expression profiles of 10 unigenes sby qRT-PCR and the correlation between the results of qRT-PCR and RNA-Seq
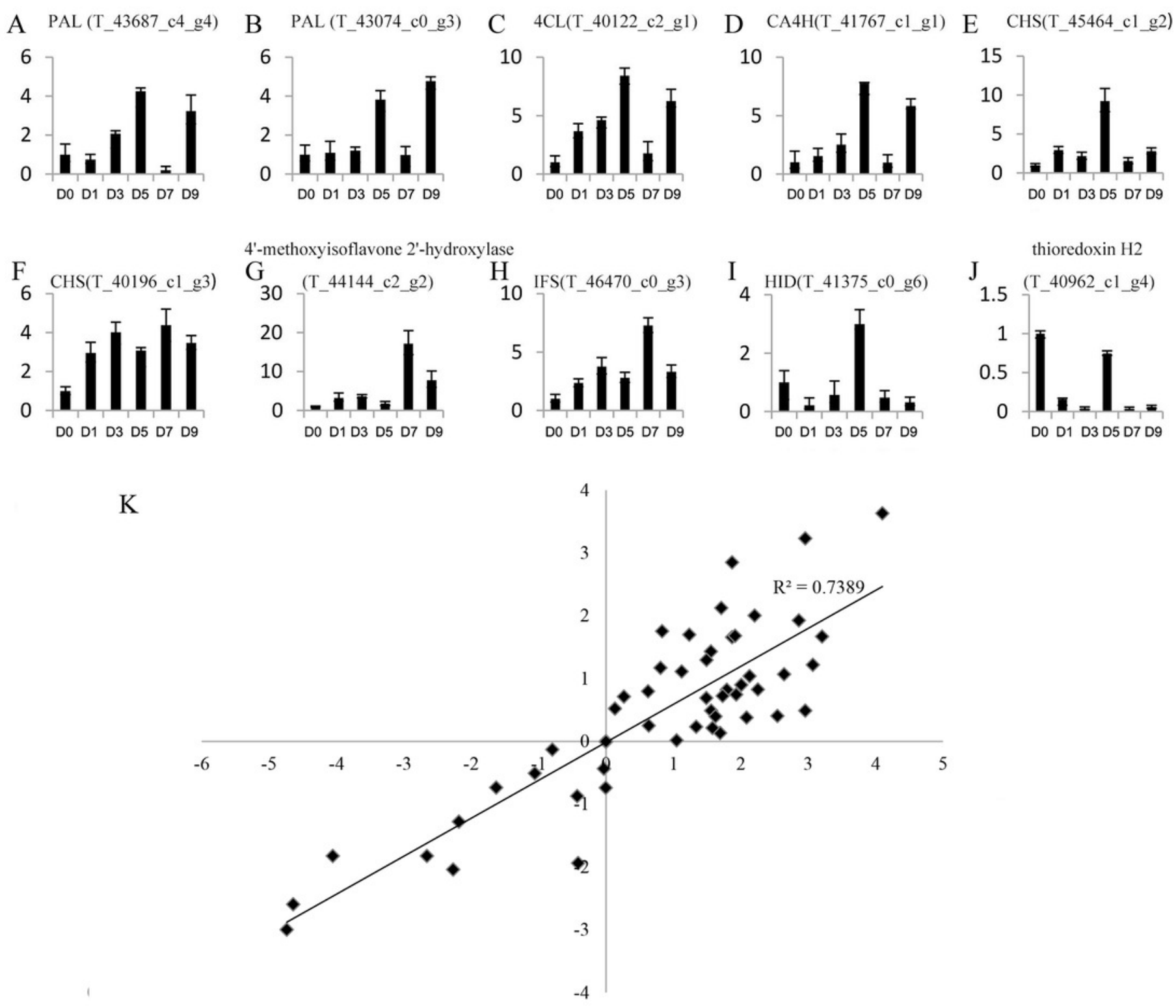
Figure 5

\section{GO enrichment of DEGs}

The $\mathrm{X}$-axis represents the enrichment factor and $\log _{10}$ of the Q-value. The $\mathrm{Y}$-axis indicates the different GO terms.

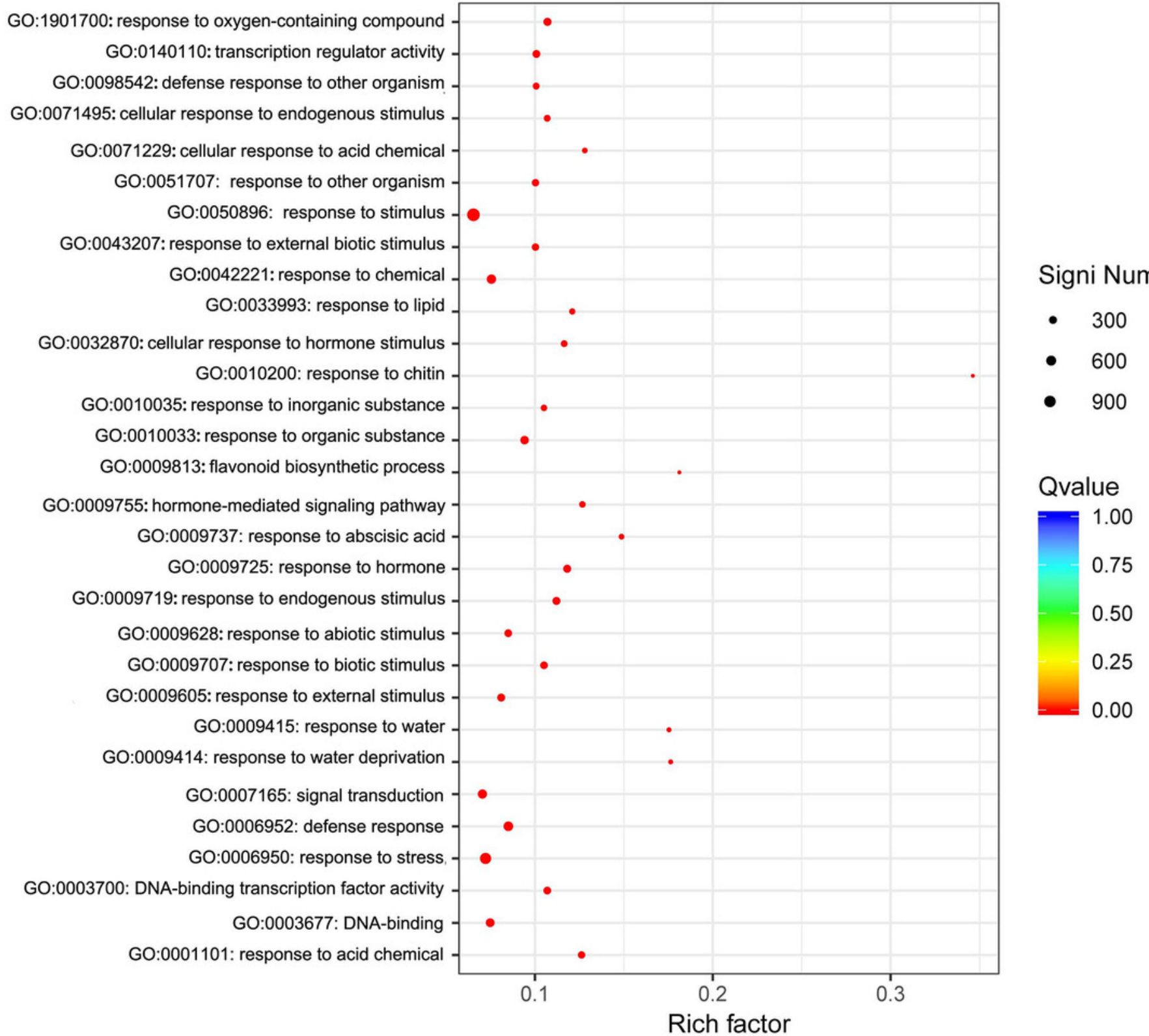


Figure 6

Representative time-course profile clusters

D0, D1, D3, D5, D7 and D9 represent differentdayaftersodiumselenitetreatment.

A

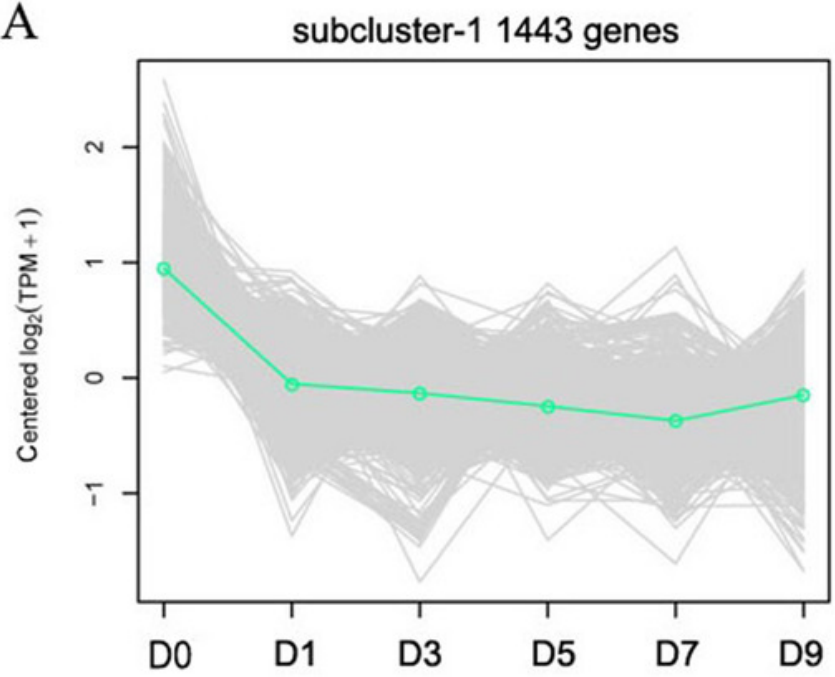

C

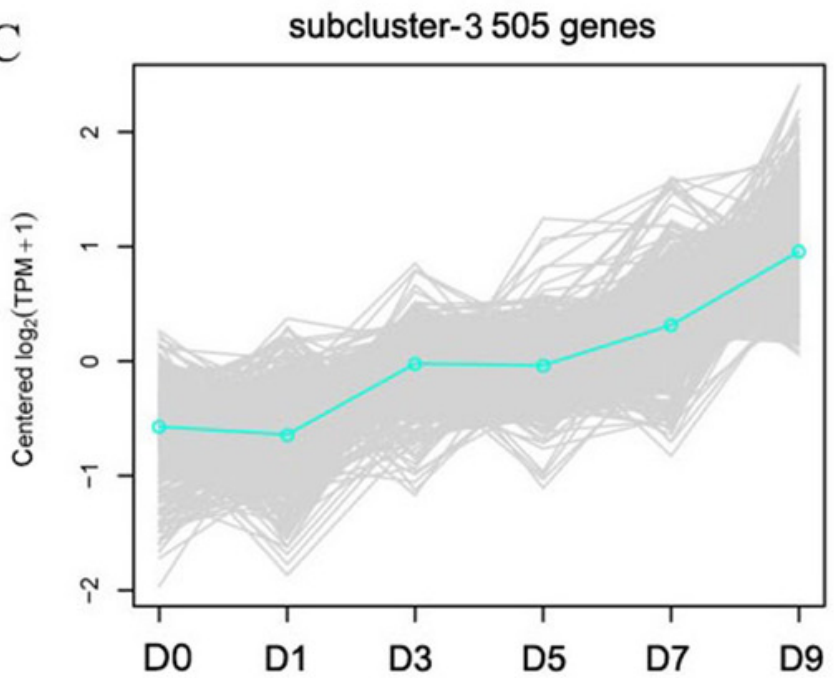

B subcluster-2 881 genes
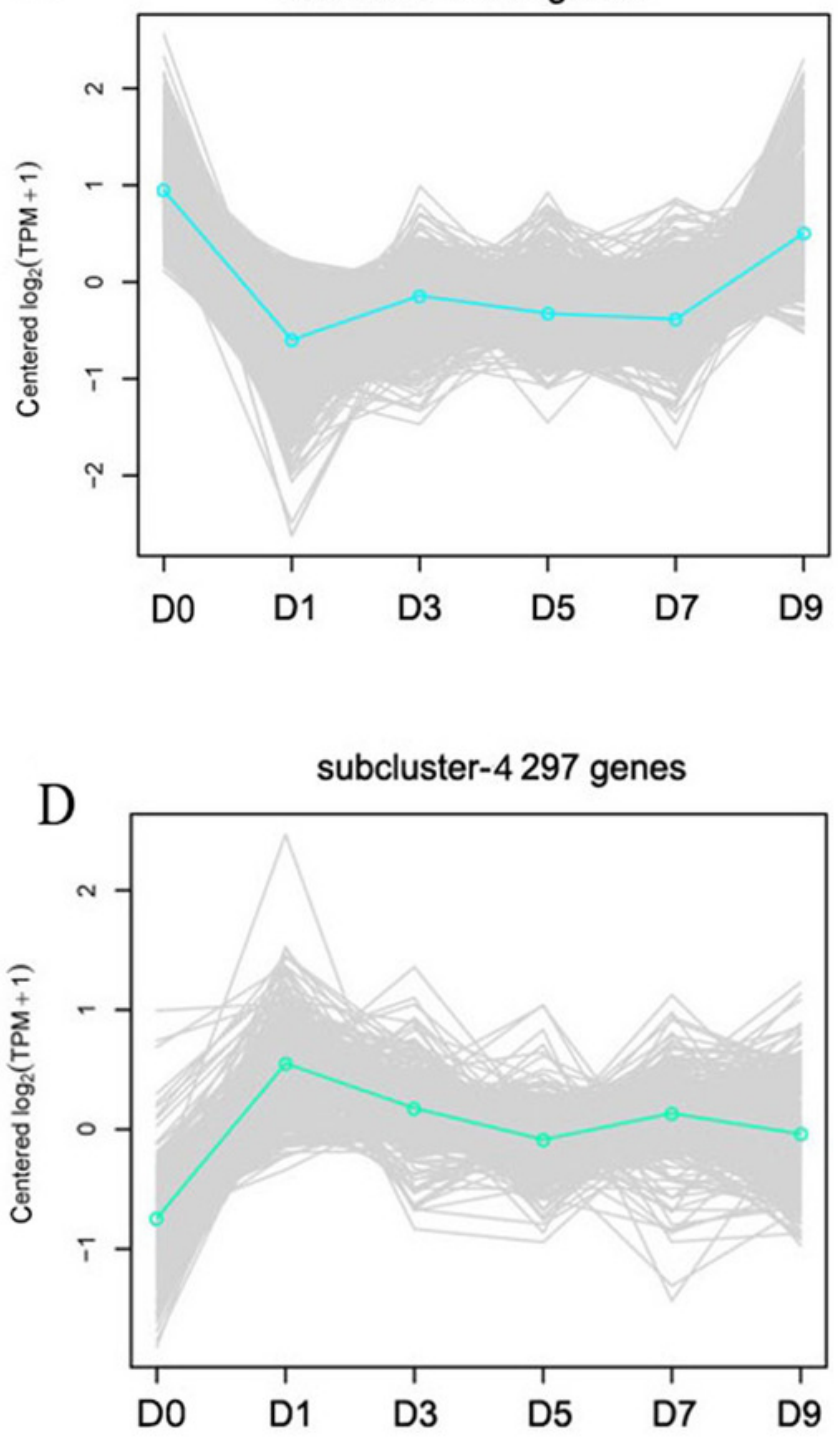


\section{Figure 7}

The overlap of the DEGs are represented by Venn diagram $(A)$ up-regulated genes and (B) down-regulated genes

A

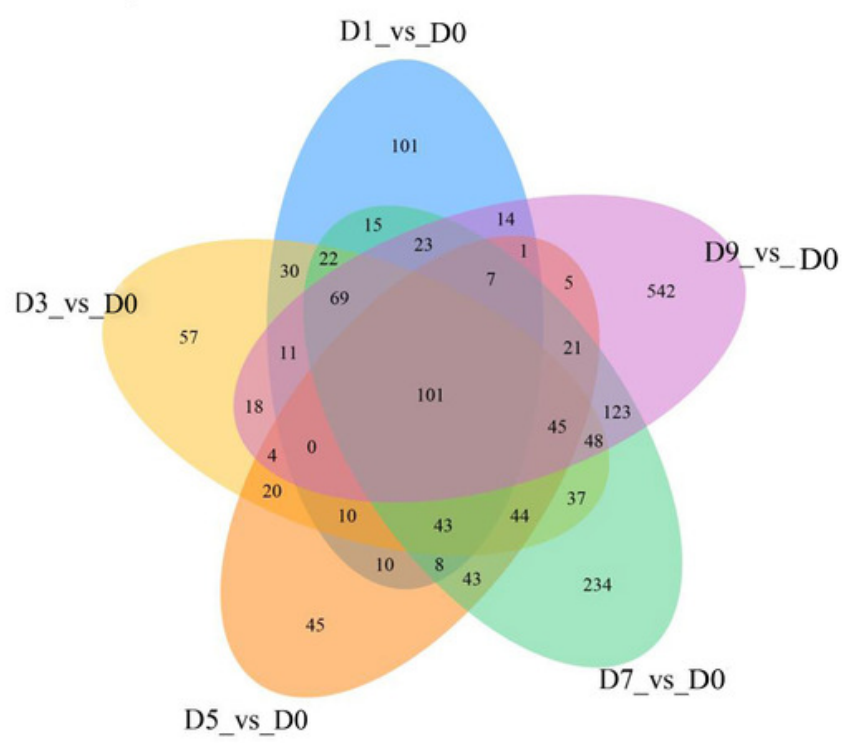

B

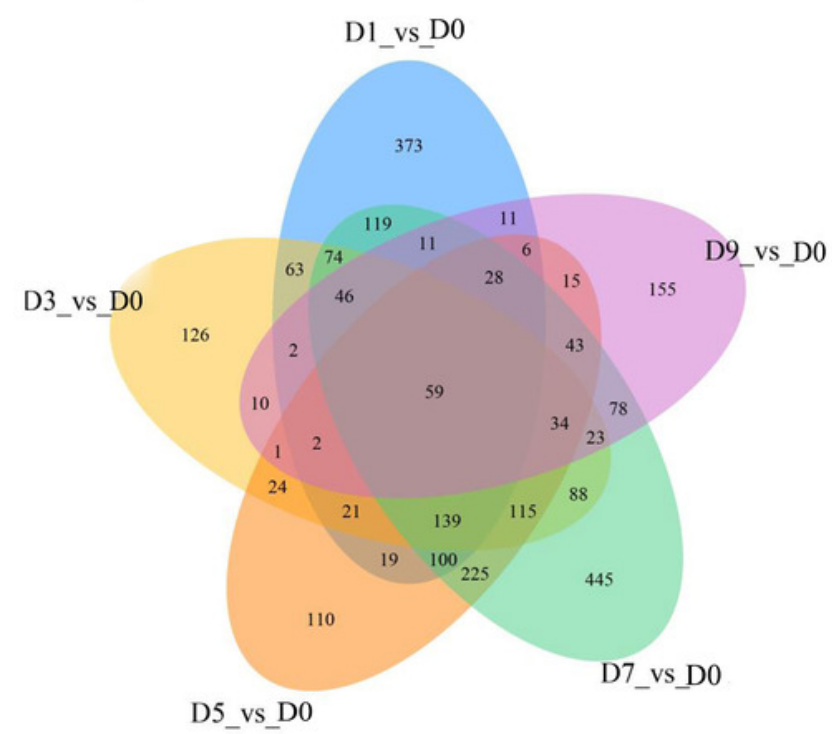

